\title{
Simultaneous Topography and Reaction Flux Mapping at and around Electrocatalytic Nanoparticles
}

\author{
Minkyung Kang, ${ }^{\dagger}$ David Perry, ${ }^{\dagger}$ Cameron L. Bentley, ${ }^{\dagger}$ Co Geoff West, ${ }^{\dagger}$ Ashley Page, ${ }^{\dagger, \S_{0}}$ \\ and Patrick R. Unwin* ${ }^{\dagger} \dagger$ \\ ${ }^{\dagger}$ Department of Chemistry, ${ }^{*}$ Warwick Manufacturing Group, and ${ }^{\S}$ MOAC Doctoral Training Centre, University of Warwick, \\ Coventry CV4 7AL, United Kingdom
}

\section{Supporting Information}

\begin{abstract}
The characterization of electrocatalytic reactions at individual nanoparticles (NPs) is presently of considerable interest but very challenging. Herein, we demonstrate how simple-to-fabricate nanopipette probes with diameters of approximately $30 \mathrm{~nm}$ can be deployed in a scanning ion conductance microscopy (SICM) platform to simultaneously visualize electrochemical reactivity and topography with high spatial resolution at electrochemical interfaces. By employing a self-referencing hopping mode protocol, whereby the probe is brought from bulk solution to the near-surface at each pixel, and with potential-time control applied at the substrate, current measurements at the nanopipette can be made with high precision and resolution ( $30 \mathrm{~nm}$ resolution, 2600 pixels $\mu \mathrm{m}^{-2},<0.3 \mathrm{~s} \mathrm{pixel}^{-1}$ ) to reveal a
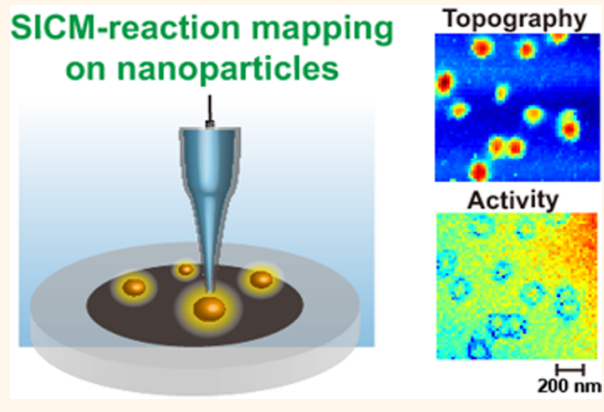
wealth of information on the substrate physicochemical properties. This methodology has been applied to image the electrocatalytic oxidation of borohydride at ensembles of AuNPs on a carbon fiber support in alkaline media, whereby the depletion of hydroxide ions and release of water during the reaction results in a detectable change in the ionic composition around the NPs. Through the use of finite element method simulations, these observations are validated and analyzed to reveal important information on heterogeneities in ion flux between the top of a NP and the gap at the NP-support contact, diffusional overlap and competition for reactant between neighboring NPs, and differences in NP activity. These studies highlight key issues that influence the behavior of NP assemblies at the single NP level and provide a platform for the use of SICM as an important tool for electrocatalysis studies.
\end{abstract}

KEYWORDS: electrochemical imaging, scanning ion conductance microscopy, electrocatalysis, nanoparticles, finite element method modeling

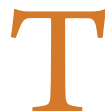
he study of catalytic nanomaterials has become an important research area, due to a range of significant real-world applications, as well as fundamental interest. ${ }^{1-4}$ Numerous studies, using ensembles of catalytic nanoparticles (NPs), have revealed that changes in NP shape, size, and structure can significantly affect (electro)catalytic activity, ${ }^{5-7}$ but investigations of activity at the individual NP level are challenging. ${ }^{8,9}$ Among a rather limited set of tools that have been applied for single NP electrochemical characterization, $^{9-14}$ scanning electrochemical probe microscopy (SEPM) techniques are particularly attractive and can be highly sensitive. ${ }^{15}$ In this paper, we demonstrate how a simple glass nanopipette can serve as a powerful and highly sensitive probe of NP size, shape, and activity, with a spatial resolution on the order of the probe size ( $30 \mathrm{~nm}$ herein).

Advances in nanoprobe fabrication and characterization procedures $^{16-18}$ have led to significant advances in several SEPMs. The widely used scanning electrochemical microscopy
(SECM) technique has recently been applied to electrocatalytic nanomaterials adhered to an electrocatalytically inert support. $^{18-20}$ However, this technique usually operates in a constant plane scanning mode with no positional feedback of the probe with respect to the surface and no topographical information obtained. Alternatively, scanning electrochemical cell microscopy (SECCM) offers integrated probe positional feedback and direct electrochemical measurements and has recently been used for the electrochemical characterization of nanomaterials (e.g., $\mathrm{sp}^{2}$ carbon nanomaterials ${ }^{21}$ and nanoparticles ${ }^{22,23}$. When applied in a correlative multimicroscopy approach, ${ }^{24,25}$ it provides a means of directly relating structure (i.e., surface structure/properties) and function (i.e., electro-

Received: July 31, 2017

Accepted: September 1, 2017

Published: September 1, 2017 

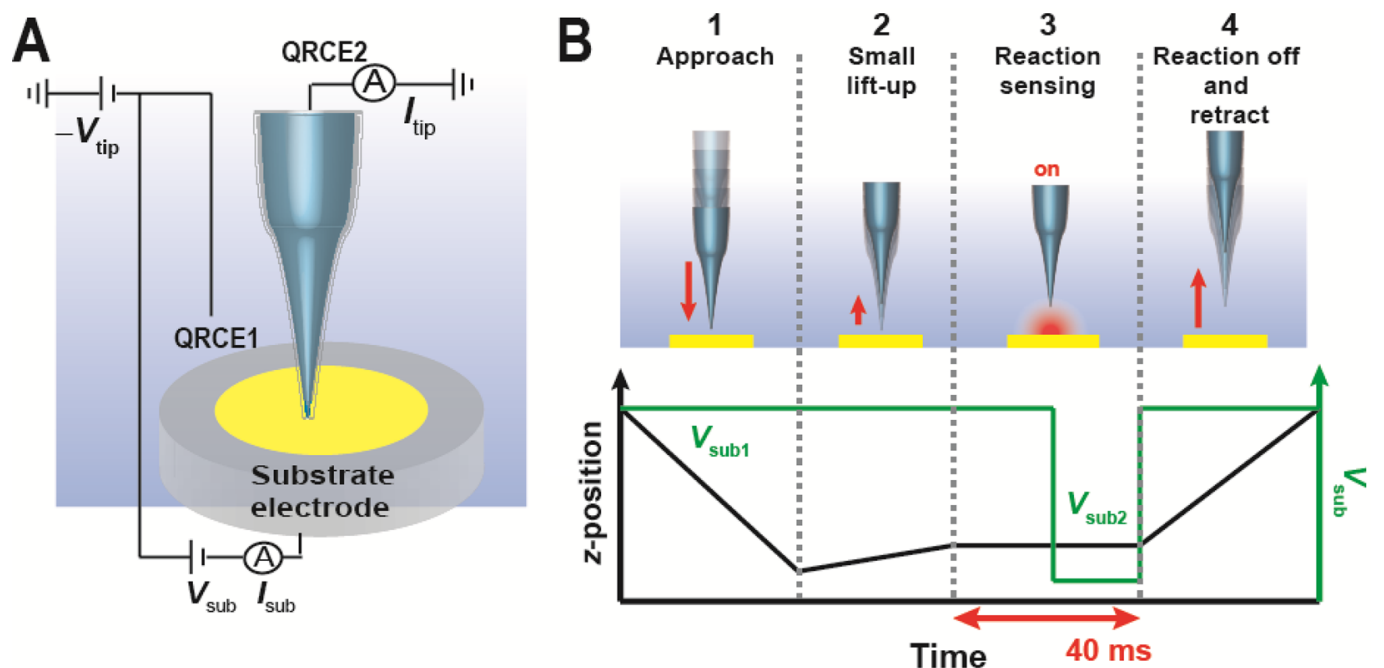

Figure 1. (A) Schematic of the experimental setup used for simultaneous topographical/electrochemical mapping of a substrate electrode using SICM. (B) Shown at the top are schematics of the main features of the imaging procedure during the hop motion of the probe (numbered 1 to 4 ) at each pixel. A trace of $z$-position and $V_{\text {sub }}$ during each step is shown at the bottom. The overall procedure can be summarized as follows: (1) approach toward the substrate surface under DC ionic current feedback to reach the set point distance; (2) small retract ("small lift-up"); (3) waiting time of $20 \mathrm{~ms}$ followed by potential step by jumping $V_{\text {sub }}$ from $V_{\text {sub1 }}$ to $V_{\text {sub2 }}$; and (4) full retract before the hop procedure is repeated at the next pixel, typically $20 \mathrm{~nm}$ lateral displacement from the previous one. The tip current, measured throughout, was analyzed as discussed in the text.

catalytic activity) at the nanoscale, which is a long-term aspiration in electrochemical science and catalysis.

To overcome the lack of positional feedback, SECM has been successfully integrated with other scanning probe techniques such as atomic force microscopy (AFM), ${ }^{26,27}$ scanning ion conductance microscopy (SICM), ${ }^{2-30}$ and scanning tunneling microscopy (STM), ${ }^{31,32}$ as well as through the use of dual redox mediators, ${ }^{33}$ to enable electrochemical and topographical images to be obtained. However, making reproducible nanoscale SECM probes is non-trivial and timeconsuming, and this can be even more challenging when multichannel probes are to be employed. ${ }^{34}$ Further, the SECM tip response needs to be stable for long time periods, and the tip current has to quantitatively detect the reactant, product, or intermediates, which becomes more challenging with the intrinsic high diffusion/migration rates at the nanoscale.

SICM is a well-established contactless topographical probe imaging technique, capable of characterizing delicate samples with nanometer scale resolution, using nanoscale glass or quartz probes that can be made very easily and quickly using a laser capillary puller. These probes are filled with electrolyte, and a contacting electrode is inserted. ${ }^{35-38}$ There has been significant progress to improve the time resolution of SICM through the use of high bandwidth electrometers and the introduction of versatile scanning regimes. ${ }^{35,39-41}$ Moreover, SICM has very recently expanded beyond its major use for topographical imaging to become a multifunctional tool capable of elucidating a variety of surface properties, beyond topography. ${ }^{15,35-38,42}$ Notably, the ion conductance current is sensitive to changes in the local ionic atmosphere near surfaces induced by either surface charge ${ }^{39,40,43}$ or as the result of electrochemical reactions. ${ }^{44}$ Since all electrochemical processes result in a change in ionic composition, SICM is potentially a very powerful general probe for visualizing nanoscale electrocatalysis.

Herein, we demonstrate how SICM with fine nanopipettes (diameter, $d_{\text {tip }} \approx 30 \mathrm{~nm}$ ) can be used to perform simultaneous electrochemical flux and topographical imaging with high spatial resolution, commensurate with the probe size. Using the electrocatalytic oxidation of borohydride $\left(\mathrm{BH}_{4}^{-}\right)$at $\mathrm{Au}$ NPs on a carbon support as an exemplar system, we show that it is possible to study and compare the electrocatalytic activity of individual NPs within an ensemble. Complementary finite element method (FEM) simulations provide insight into several critically important phenomena such as the competition between neighboring NPs for reactant, and heterogeneities in ion fluxes around a single NP. This study highlights SICM as a key tool for mapping nanoscale electrochemical processes and provides a roadmap for future applications of SICM in this area.

\section{RESULTS AND DISCUSSION}

Electrochemical SICM Operating Principles. Salient experimental details are given in the Methods section at the end of the paper, but to understand the approach, it is necessary to outline key features of the SICM protocol developed. The strategy implemented allows a detailed correlation between structure (topography) and function (electrochemical reactivity) at the nanoscale. Figure 1A provides a schematic illustration of the experimental setup. ${ }^{44}$ Briefly, electrochemical control of the substrate (working) electrode potential was achieved with a potential $V_{\text {sub }}$ (variable) applied with respect to a quasi-reference counter electrode (QRCE) in the bulk electrolyte/reactant solution, denoted QRCE1, producing the substrate current $\left(I_{\text {sub }}\right)$. QRCE1 was biased at a potential of $-V_{\text {tip }}$ with respect to ground, for the ion conductance measurements with the SICM tip, via QRCE2 (at ground) inside a quartz nanopipette (filled with the same solution as the bulk; vide infra). The bias, $V_{\text {tip }}$, resulted in an ion conductance current $\left(I_{\text {tip }}\right)$, which was monitored throughout the scanning process and was used to both position the tip above the substrate (i.e., to obtain topographical data) and probe ion fluxes in the vicinity of the surface induced by an electrochemical reaction (i.e., electrocatalytic activity data). A representative transmission electron microscopy (TEM) image 
of a nanopipette probe is shown in Supporting Information, Figure S1. It has an inner diameter of $\approx 30 \mathrm{~nm}$.

A direct current (DC) hopping mode with self-referencing was implemented. $I_{\text {tip }}$ was measured throughout the entire imaging process every $4 \mu \mathrm{s}$ with 129 points averaged to give a data point every $516 \mu \mathrm{s}$, as the probe was approached from bulk solution to the surface at each lateral position (pixel). ${ }^{29,39}$ When surface charge (double layer) effects are negligible, $I_{\text {tip }}$ decreases appreciably as the tip is approached within a distance of about one tip diameter from the surface. ${ }^{38}$ This was a reasonable consideration for the studies herein, given the electrolyte concentration, tip/substrate separation, and $V_{\text {sub }}$ of 0 $\mathrm{V} v s \mathrm{Pd}-\mathrm{H}_{2} \mathrm{QRCE}$ on approach, which is close to the potential of zero charge of $\mathrm{Au}$ at high $\mathrm{pH} .{ }^{45} \mathrm{~A}$ feedback threshold, or set point, of $I_{\text {tip }}$ corresponding to a decrease of $4.1 \%$ between the bulk and surface was set, and the tip-substrate separation distance on approach was estimated from the measured $I_{\text {tip }}$ value, which was used in FEM simulations to estimate the actual separation distance (see Supporting Information, sections S2 and S3). For surfaces that were planar on the scale of the nanopipette probe opening, that is, over the UME and even over the top of nanostructures, the final $I_{\text {tip }}$ value herein, during approach, corresponded to a closest tipsubstrate separation distance of approximately $6 \mathrm{~nm}$, slightly closer to the surface than the target due to the intrinsic response latency of the piezo and control system. ${ }^{35}$ This end position of the nanopipette at each approach ( $z$-extension) was used to construct high-resolution 3D topographical maps (e.g., Figure 1B, part 1). It is important to note that SICM approach curves to a curved or tilted surface (e.g., over a NP edge) would yield a different separation distance than those performed over a flat surface. Such a situation is quite rare in an image but is still taken into account in FEM simulations performed, as the working distance is calculated based on the experimental final value of $I_{\text {tip }}$. For clarity, where probe-substrate separation distances are quoted herein, these correspond to the distance that would be achieved above the top of a nanostructure or planar surface.

Upon detecting the near surface (reaching the target set point), the nanopipette immediately retracted a fixed distance (vide infra), which we call a "small lift-up" (typically $20 \mathrm{~nm}$, but varied in some experiments), a distance at which the measurements of electrochemical activity were obtained (Figure 1B, part 2). This step was implemented to (i) minimize the impact of the probe on mass transport at the substrate (i.e., physical blockage of the surface reaction flux by the tip); (ii) minimize any effects of the electrical double layer on the recorded response, especially when rapidly changing the substrate potential with time (vide infra); while (iii) maintaining high spatial and time resolution for electrochemical image acquisition, by measuring electrocatalytic ion fluxes at sufficiently close distances to the surface (vide infra). The distance between the tip and the substrate during the electrochemical measurement $\left(d_{s-t}\right)$ is the sum of the estimated tip distance from the initial approach curve (i.e., $6 \mathrm{~nm}$ ) and the distance of "small lift-up" set by the operating program.

After lift-up, a potential step of the $V_{\text {sub }}$ was applied after a waiting period of $20 \mathrm{~ms}$. The substrate potential was jumped from $V_{\text {subl }}$, where no (electrochemical) reaction occurred at the substrate, to $V_{\text {sub2 }}$, where the electrocatalytic reaction was "switched on", typically for just $20 \mathrm{~ms}$ at each pixel (see Figure $1 \mathrm{~B}$, part 3). This is an advantageous feature of this technique, serving to reduce any possible deterioration in the surface activity that could be caused by prolonged turnover of the reactant on the catalyst surface. ${ }^{46,47}$ For the maps of electrochemical activity presented herein, up to 3136 pixels were recorded, meaning that the reaction was only "on" for about $1 \mathrm{~min}(62.72 \mathrm{~s})$. On this time scale, there was typically only $4 \%$ random variation in $I_{\text {sub }}$ with respect to the average $I_{\text {sub }}$ when the reaction was "on". For the data presentation in the images herein, $I_{\text {tip }}$ measured when the substrate reaction was "on" was normalized with respect to $I_{\text {tip }}$ when reaction was "off". This is denoted by $I_{\text {tip }}\left(V_{\text {sub2 } 2}\right) / I_{\text {tip }}\left(V_{\text {sub1 }}\right)$ and referred to as "normalized $I_{\text {tip }}$ " throughout. $I_{\text {tip }}\left(V_{\text {sub1 }}\right)$ was the average value of the last $5 \mathrm{~ms}$ at $V_{\text {sub1 }}$ before the step in potential to $V_{\text {sub2 }}$ and $I_{\text {tip }}\left(V_{\text {sub2 }}\right)$ was measured over the last $5 \mathrm{~ms}$ of the $20 \mathrm{~ms}$ potential step to minimize any influence of non-faradaic (charging) and non-steady-state effects at short times. Note that the substrate reaction is not at true steady state during this period but tends toward this situation (vide infra). Some transient tip current data are also presented, such as in Supporting Information, Figure S2, which gives an example $I_{\text {tip }}$-time trace, together with the corresponding change in tipsubstrate distance and $V_{\text {sub }}$ during a single pixel measurement.

Prior to approach at the next pixel, the nanopipette was rapidly withdrawn into bulk to a distance between $400 \mathrm{~nm}$ and $1.2 \mu \mathrm{m}$, depending on the sample roughness (Figure 1B, part 4). Overall, the procedure resulted in a pixel acquisition time of around $0.3 \mathrm{~s} \mathrm{pixel}^{-1}$ (see Supporting Information, Figure S2), allowing relatively high electrochemical (and topographical) image acquisition (mapping) rates: typical scans of 2600 pixels $\mu \mathrm{m}^{-2}$ (3136 pixels in a scan area $\approx 1.2 \mu \mathrm{m}^{2}$ ) took approximately $15 \mathrm{~min}$. It is important to note that while higher imaging scan rates are certainly possible in SICM experiments, ${ }^{35,44,48}$ these typically only consider SICM for topographical measurements. The inherent nature of making functional (electrochemical) measurements with SICM means that, in most cases, longer exposure times of the tip near the surface are required.

Electrochemical Mapping on UMEs. $\mathrm{BH}_{4}^{-}$electrooxidation on an Au surface in alkaline media was selected as the model electrocatalytic process. $\mathrm{NaBH}_{4}(3 \mathrm{mM})$ with 30 $\mathrm{mM} \mathrm{NaOH}(\mathrm{pH}$ 12.5) electrolyte solution was used for the experiments to ensure that the $\mathrm{pH}$ was greater than 12 and that the $\left[\mathrm{OH}^{-}\right] /\left[\mathrm{BH}_{4}^{-}\right]$ratio was greater than 4.4 , which has been shown to suppress the chemical hydrolysis process, which consumes $\mathrm{BH}_{4}^{-}$and releases $\mathrm{H}_{2}{ }^{49,50}$ The net reaction for borohydride electro-oxidation is as follows:

$$
\mathrm{BH}_{4}^{-}+8 \mathrm{OH}^{-} \rightarrow \mathrm{BO}_{2}^{-}+6 \mathrm{H}_{2} \mathrm{O}+8 e^{-}
$$

where for each $\mathrm{BH}_{4}{ }^{-}$oxidized, $8 \mathrm{OH}^{-}$ions are consumed from the electrolyte in an overall 8-electron process. This model inner-sphere reaction is known to be sensitive to the type ${ }^{46,51}$ and surface crystallographic orientation of the metal electrode. ${ }^{7,52}$ Additionally, it is worth noting that this reaction is of practical importance, as it is the anodic process in direct borohydride fuel cells. ${ }^{46,51}$

Linear sweep voltammetry in the configuration shown in Figure 1, performed at an Au ultramicroelectrode (UME) substrate $(\approx 10 \mu \mathrm{m}$ diameter $)$, with $\mathrm{Pd}-\mathrm{H}_{2}$ QRCE1, showed the characteristic $\mathrm{BH}_{4}{ }^{-}$electro-oxidation response expected in alkaline media (Figure 2A,B, black solid line). In agreement with previous studies, ${ }^{53,54}$ the substrate current begins to increase near a potential of $0.1 \mathrm{~V}$ due to the oxidation of $\mathrm{BH}_{4}^{-}$, reaching a plateau in the potential range from 0.6 to $1.1 \mathrm{~V}$. Note that the linear sweep voltammograms (LSVs) shown in Figure $2 \mathrm{~A}, \mathrm{~B}$ were measured on different $\mathrm{Au}$ UMEs at different 

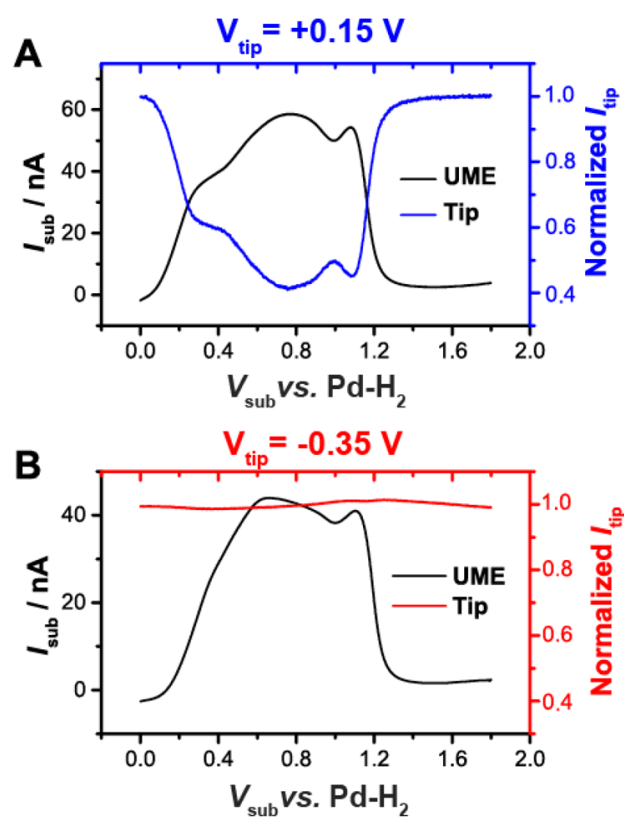

C

Competition mode Bottleneck effect

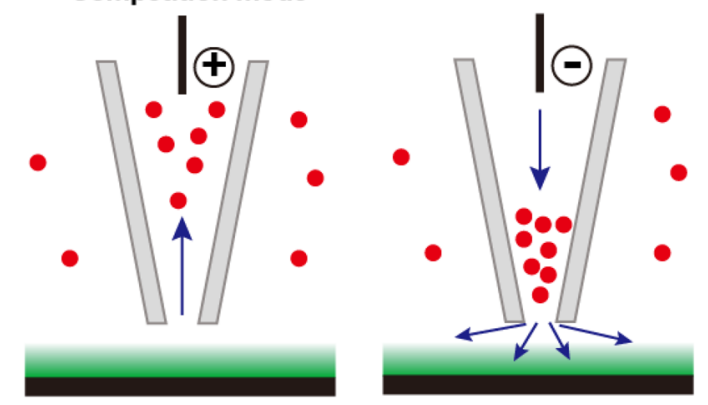

- OH-ion accumulation

$\mathrm{OH}$ - ion flux due to the tip bias

$\mathrm{OH}$ - ion depletion due to the reaction at the surface

Figure 2. LSVs (potential sweep at $V_{\text {sub }}$ ) obtained at Au UMEs (diameter $\approx 10 \mu \mathrm{m}$ ) in a solution containing $3 \mathrm{mM} \mathrm{NaBH}_{4}$ and 30 $\mathrm{mM} \mathrm{NaOH}$ with a scan rate of $0.2 \mathrm{~V} \mathrm{~s}^{-1}$. The substrate current $\left(I_{\text {sub }}\right)$ and tip current $\left(I_{\text {tip }}\right)$ were concurrently measured at fixed tip bias potentials $\left(V_{\text {tip }}\right)$ of $(\mathrm{A})+0.15 \mathrm{~V}$ and $(\mathrm{B})-0.35 \mathrm{~V}$. The SICM probe was positioned at the center of the Au UME at a vertical distance of $26 \mathrm{~nm}$ (vide infra) during the measurements. For normalization, the magnitude of $I_{\text {tip }}$ without the substrate reaction was (A) $180 \mathrm{pA}$ and (B) $-850 \mathrm{pA}$. (C) Schematics (not to scale) illustrating $\mathrm{OH}^{-}$ion accumulation and changes in the $\mathrm{OH}^{-}$flux near the end of the nanopipette at positive (left) and negative (right) tip biases during $\mathrm{BH}_{4}^{-}$oxidation.

times, resulting in the slight difference in the magnitude of the oxidation current. At a potential of $1.1 \mathrm{~V}$, an $\mathrm{AuO}_{x}$ monolayer starts to form on $\mathrm{Au}$, passivating the surface and "switching off" the $\mathrm{BH}_{4}^{-}$oxidation reaction, resulting in a rapid decrease in current with the ongoing anodic potential scan. ${ }^{53,54}$ As the voltage was swept further positive (from 1.4 to $1.8 \mathrm{~V}$ ), the substrate current again began to slowly increase, attributable to the gradual formation of an $\mathrm{AuO}_{x}$ multilayer. ${ }^{55}$

$I_{\text {tip }}$ was also monitored simultaneously during the voltage sweep at two different tip-electrode potential biases (QRCE2), $V_{\text {tip }}$, with respect to QRCE1. For this purpose, a nanopipette was centered on the Au electrode with $d_{s-t}$ of $26 \mathrm{~nm}$ (Figure 2A,B, blue and red solid lines). When the QRCE2 (in the tip) was biased positively at $0.15 \mathrm{~V}$ (relative to QRCE1 in bulk) throughout the substrate voltage sweep, $I_{\text {tip }}$ was found to be extremely sensitive to the substrate electrochemical reaction (Figure 2A). The consumption of $\mathrm{OH}^{-}$at the $\mathrm{Au}$ substrate leads to a significant change in ionic strength near the surface that is manifested as a decrease in normalized $I_{\text {tip }}$, which tracks the changes in $I_{\text {sub }}$ very faithfully. In contrast, when the tip was biased negatively at $-0.35 \mathrm{~V}, I_{\text {tip }}$ was seen to be insensitive to the substrate reaction (Figure $2 \mathrm{~B}$ ). ${ }^{56}$ Note that the bias on the tip has little influence on the substrate UME voltammetric characteristics. This was also confirmed by measuring the voltammetric behavior without the SICM tip present, which was as reported in the previous study. ${ }^{54}$ Note that although QRCE2 could, in principle, act as a counter electrode for the substrate reaction (especially when biased negative with respect to QRCE1), the narrow dimensions of the nanopipette means that this is a very resistive path, and so the counter current for the substrate reaction flows through QRCE1. These initial experiments demonstrate that the SICM tip is a relatively noninvasive probe of the substrate reaction, but one whose sensitivity depends on the tip bias.

FEM simulations were carried out to rationalize the experimental observation that $I_{\text {tip }}$ was most sensitive to the substrate reaction with a positively biased tip. The surface charge on the nanopipette was estimated to be approximately $-40 \mathrm{mC} \mathrm{m}^{-2}$, due to the high solution $\mathrm{pH}$ (see Supporting Information, section S3, Figure S3). ${ }^{57}$ As summarized in the schematic in Figure 2C, when the tip is positively biased (Figure $2 \mathrm{C}$, left), $\mathrm{OH}^{-}$ions (the major current carrying ion) are depleted near the end of the tip due to migration toward QRCE2 in the tip, a process that is in competition with the depletion in $\mathrm{OH}^{-}$that occurs in the vicinity of the substrate when the electrochemical reaction is switched on. By contrast, with a negatively biased tip (Figure $2 \mathrm{C}$, right), $\mathrm{OH}^{-}$ions accumulate near the end of the tip, due to an asymmetry in mass transport through the tip (slower) and away from the end (faster). This produces a "bottleneck effect", so that the conductance of the tip end (the most resistive part of the circuit) is similar whether the substrate reaction is on or off. Consequently, the normalized $I_{\text {tip }}$ value is close to unity during the scan of the substrate electrode potential, as seen in Figure $2 \mathrm{~B}$.

The FEM simulation results presented in the Supporting Information, Figure S5, confirm these findings: at positive tip bias, the $\left[\mathrm{OH}^{-}\right]$profile and overall ion current reaction in the tip (and hence current in the SICM circuit) change between a slow and fast substrate reaction, whereas at negative tip bias, the ionic concentration at the tip end is enhanced but is essentially invariant with the rate of the substrate reaction. For reaction imaging, a positive tip bias was thus used herein, unless stated otherwise.

Tip Distance Effects in Reaction Mapping. It was important to assess what the tip measures at different distances from the substrate and to establish the region where the tip is mainly sensitive to the substrate electrode reaction. In addition to the reactive ion fluxes as considered herein, the ionic current in SICM may be sensitive to the electrical double layer at charged substrates. ${ }^{39,40,43}$ For electrochemical imaging, a large positive bias is applied at the substrate electrode to drive $\mathrm{BH}_{4}^{-}$ electro-oxidation, and therefore, the surface charge will be more positive than when the reaction is off $\left(V_{\text {sub }}=0 \mathrm{~V}\right.$ relative to the bulk QRCE). Based on previous SICM surface charge studies, ${ }^{39,40,43,58,59}$ at positive tip bias, this would be expected to result in an enhanced tip current, whereas the tip current 

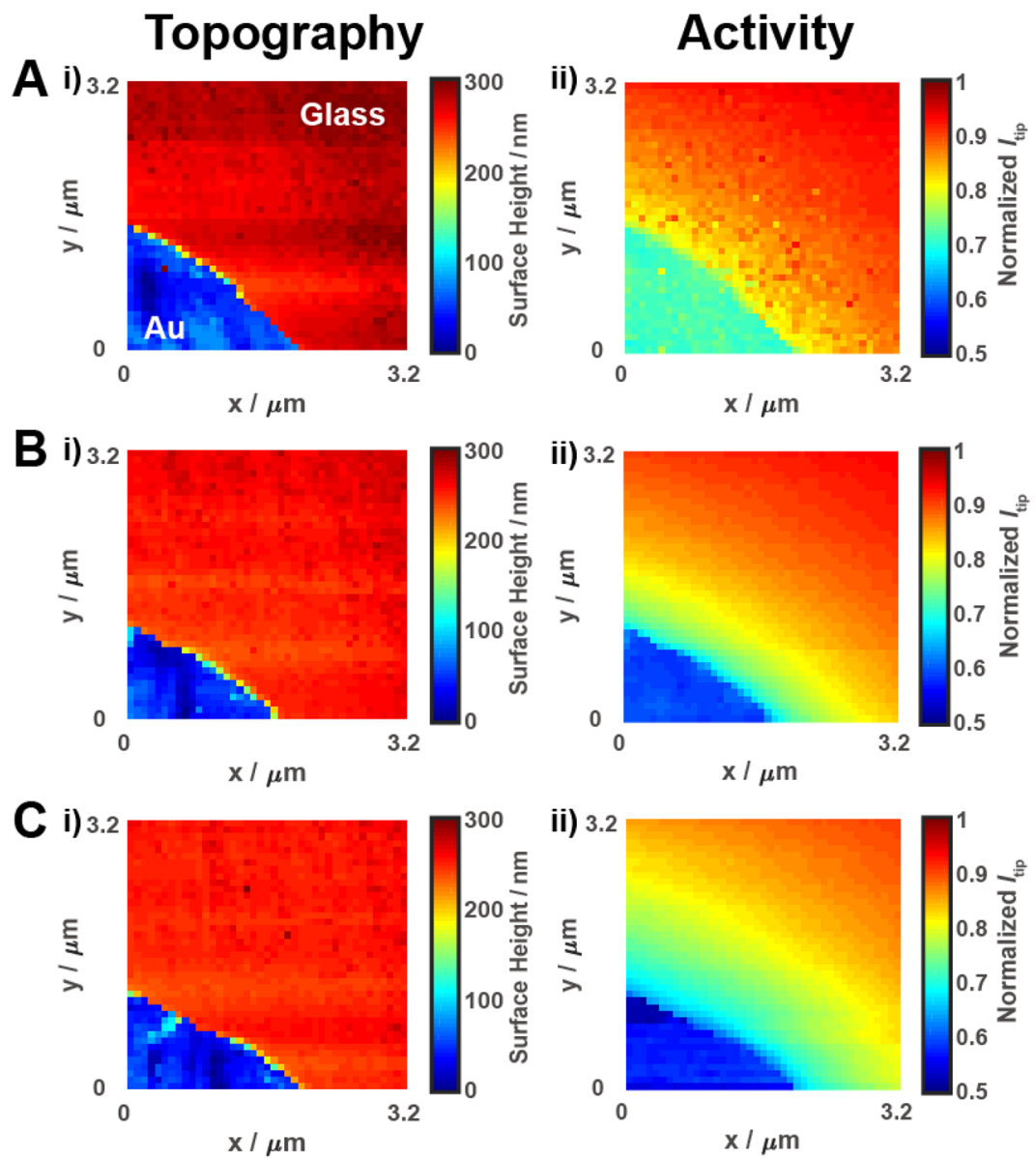

Figure 3. (i) SICM topography and (ii) electrochemical activity maps of an Au UME (diameter $\approx 10 \mu \mathrm{m}$ ), obtained simultaneously, in a solution containing $3 \mathrm{mM} \mathrm{NaBH}_{4}$ and $30 \mathrm{mM} \mathrm{NaOH}$, recorded with $V_{\text {sub2 }}=0.9 \mathrm{~V}, V_{\text {tip }}=+0.15 \mathrm{~V}$, and $d_{\mathrm{s}-\mathrm{t}}$ values of $(\mathrm{A}) 11 \mathrm{~nm},(\mathrm{~B}) 26 \mathrm{~nm}$, and (C) $106 \mathrm{~nm}$. There is no interpolation of the data, and each of the images contains 1681 pixels.

decreases when the substrate reaction is on (Figure $2 \mathrm{~A}$ ). The effect of surface charge is further extinguished the greater the tip-substrate separation. ${ }^{58,59}$ In contrast, the change in ionic composition due to the substrate reaction extends over the concentration (diffusion) boundary layer, which is orders of magnitude larger. Thus, one can set a "near interface distance" for reaction imaging, where there is a significant change in ionic strength from the substrate reaction but where the tip is insensitive to surface charge effects. Furthermore, situating the tip a little further from the substrate is also advantageous in minimizing the extent to which mass transport to the substrate is blocked by the tip. ${ }^{60}$

To investigate the effect of $d_{s-t}$ on the SICM response, electrochemical and topographical images of an Au UME and surrounding glass sheath were obtained at $d_{s-\mathrm{t}}$ values of 11,26 , and $106 \mathrm{~nm}$, with the resulting maps for $V_{\text {sub2 }}=0.9 \mathrm{~V}$ (pixel density of 165 pixels $\mu \mathrm{m}^{-2}$ ) shown in Figure $3 \mathrm{~A}-\mathrm{C}$, respectively. The topographical maps are remarkably consistent and highlight an Au electrode that is recessed by about $300 \mathrm{~nm}$ compared to the surrounding glass sheath, resulting from the polishing process during UME fabrication/conditioning. In contrast, the activity maps are much more distance-dependent. Notably, the activity map obtained at a $d_{s-t}$ of $11 \mathrm{~nm}$ (Figure $3 \mathrm{~A})$ shows a much diminished change in normalized $I_{\text {tip }}$ between the $\mathrm{Au}$ substrate and glass sheath, when compared to the larger $d_{s-t}$ values of $26 \mathrm{~nm}$ and even $106 \mathrm{~nm}$ (Figure $3 \mathrm{~B}, \mathrm{C})$. This could, in part, be due to the tip sensing the electrical double layer at such small probe-substrate separations (as discussed above) and/or physical blockage of the reaction flux at the surface by the tip, as is well-known in other local probe measurements of electrochemical interfaces. ${ }^{60,61}$ In contrast, when $d_{s-t}$ is relatively large (Figure 3C), there is a much shallower lateral gradient in $I_{\text {tip }}$ between the active electrode and glass insulator compared to $d_{\mathrm{s}-\mathrm{t}}=26 \mathrm{~nm}$. Consequently, a balance needs to be struck between $d_{s-t}$ being small enough to provide the desired resolution but large enough to avoid the effects seen in Figure $3 \mathrm{~A}$. As a result, $d_{s-\mathrm{t}}$ was fixed at $26 \mathrm{~nm}$ for the experiments discussed further below. Additional simultaneously recorded electrochemical and topographical images obtained at $d_{\mathrm{s} \text {-t }}=26 \mathrm{~nm}$, with $V_{\text {sub2 }}$ set to be either 0.3 or $0.6 \mathrm{~V}$ (Supporting Information, Figure S6), further demonstrate how the proposed SICM approach is sensitive to the reaction rate at the UME substrate, an aspect that we now develop next for single NP mapping.

Single NP Mapping. In contrast to $\mathrm{Au}$ electrodes, carbon fiber (CF) is an electrocatalytically inert material for $\mathrm{BH}_{4}^{-}$ electro-oxidation ${ }^{47,62}$ (see Supporting Information, Figure S7A). $I_{\text {tip }}\left(V_{\text {tip }}=+0.15 \mathrm{~V}\right)$ maps of a CF UME showed no contrast between the carbon and glass surfaces, with uniform values of normalized $I_{\text {tip }}$ close to one, in the potential range of interest, as expected for an inert substrate (see Supporting Information, Figure S7B,C). CF was thus a suitable support material to explore electrocatalytically active individual Au NPs and nanostructures. A CF UME with Au nanostrutures was 


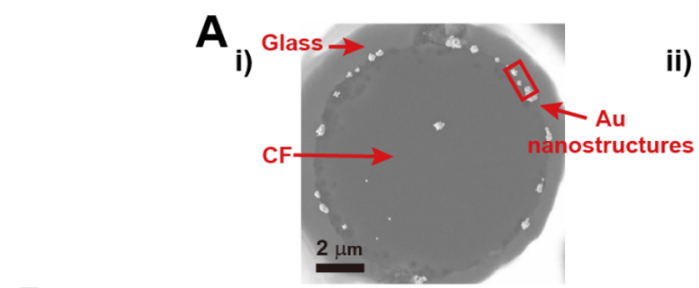

B
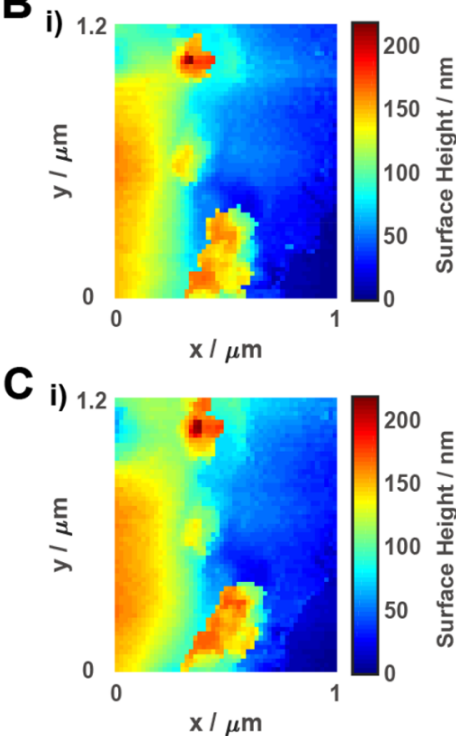

ii)

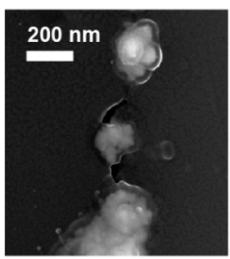

iii)
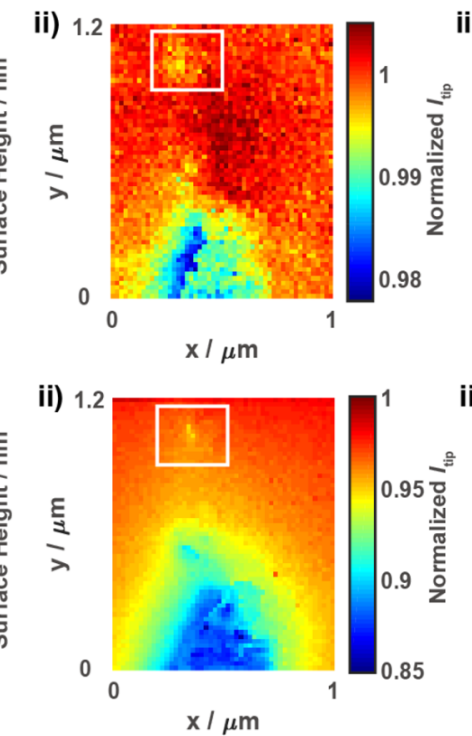

iii)

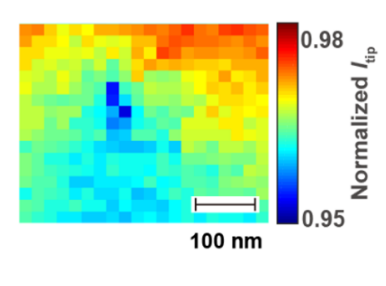

Figure 4. (A) SEM images of Au nanostructures on a CF UME: (i) electron micrograph of the CF UME with deposited Au nanostructures and (ii) magnified view of the red box in (i), indicating the area imaged with SICM. (B) (i) SICM topography map and (ii) corresponding electrochemical activity image of Au nanostructures at $V_{\text {sub2 }}=0.65 \mathrm{~V}$. (iii) Replotted electrochemical image of selected area in B(ii), with the scale bar indicating $100 \mathrm{~nm}$. (C) Identical to (B), except the data were obtained at a $V_{\text {sub2 }}$ of $0.9 \mathrm{~V}$. Data were obtained in a solution containing $3 \mathrm{mM} \mathrm{NaBH}$ and $30 \mathrm{mM} \mathrm{NaOH}$ at $V_{\text {tip }}$ of $+0.15 \mathrm{~V}$, recorded with a $d_{\text {s-t }}$ value of $26 \mathrm{~nm}$ (when the nanopipette is positioned above the top of the particle). There is no interpolation of the data, and the full SICM images contain 3111 pixels in total. Note that the SICM image shows the CF to protrude from the surrounding glass sheath (raised areas in the left of the topographical images).

prepared as described in the Methods section. The scanning electron microscopy (SEM) images in Figure 4A show that $\mathrm{Au}$ nanostructures were mostly deposited at the boundary between the CF and the glass sheath of the UME and that the size of the nanostructures varied from 150 to $800 \mathrm{~nm}$, with different shapes resulting from the aggregation of small $(10-50 \mathrm{~nm}) \mathrm{Au}$ clusters. Comparison of the topographical maps [Figure $4 \mathrm{~B}, \mathrm{C}(\mathrm{i})]$, obtained as part of the SICM protocol, with the SEM images [Figure 4A(ii)] shows that SICM topographical imaging (pixel density of 2600 pixels $\mu \mathrm{m}^{-2}$ ) provides size information in situ at a resolution and accuracy that is comparable to electron microscopy for the range of scale of the nanostructures studied herein.

Two typical electrochemical activity images with $V_{\text {sub2 }}$ of 0.65 and $0.9 \mathrm{~V}$ are shown in Figure $4 \mathrm{~B}(\mathrm{ii}), \mathrm{C}(\mathrm{ii})$, respectively. These maps are just $1 \times 1.2 \mu \mathrm{m}^{2}$ and comprise 3111 pixels. The magnitude of the change in normalized $I_{\text {tip }}$ during the electrochemical reaction on the Au nanostructures is much smaller than observed on the Au UME under comparable conditions, evident by comparing the normalized $I_{\text {tip }}$ values in Figure $3 \mathrm{~B}$ and Figure $4 \mathrm{C}$. This is because the mass transport rate to the $\mathrm{Au}$ nanostructures is considerably higher than that for the Au UME, and so there are greater kinetic limitations at the nanostructures at the same driving force. This aspect is further evident from Figure 4B,C, where it can be seen that the larger $\mathrm{Au}$ nanostructures (bottom, approximately $800 \mathrm{~nm}$ largest dimension) give rise to a more significant decrease in $I_{\text {tip }}$ compared to two smaller $\mathrm{Au}$ nanostructures (middle and top, approximately 150 and $200 \mathrm{~nm}$ ) as a result of greater depletion of the interfacial ion concentration of $\mathrm{BH}_{4}^{-}$and $\mathrm{OH}^{-}$at the larger structure.

It is important to highlight that considerable detail on local nanostructure activity can be obtained due to the high pixel density. Although there is overlap of diffusion between neighboring nanostructures, particularly at higher substrate potential, such that the activity of the large lower nanostructure dominates, it is still possible to pinpoint the active top NP, for example, part of Figure 4C(ii) shown in Figure 4C(iii) and its activity quite easily seen at lower substrate driving force [part of Figure 4B(ii) shown in Figure 4B(iii)].

A more homogeneous distribution of AuNPs on a CF UME was produced as outlined in the Methods section. SEM and SICM images of the resulting AuNPs are shown in Figure 5. Cross-sectional TEM images of individual AuNPs confirmed an ellipsoidal 3-D shape (see Supporting Information, Figure S8), such that there was a small gap between the AuNP and the CF substrate support. The average height of the AuNPs $\left(h_{\mathrm{NP}}\right)$ characterized by cross-sectional TEM imaging was $76 \pm 16 \mathrm{~nm}$ $(N=7)$. Analysis of the size distribution of the AuNPs by SEM imaging [Figure $5 \mathrm{~A}(\mathrm{i})$ ], yielded average diameters $\left(d_{\mathrm{NP}}\right)$ of 125 $\pm 15 \mathrm{~nm}(N=160)$. The size distribution in the area of interest (vide infra) yielded an average $d_{\mathrm{NP}}$ of $128 \pm 17 \mathrm{~nm}(N=11)$ [SEM image in Figure 5A(ii), AuNPs in red box]. Individual particles are annotated (Figure 5B) for further analysis and discussion below. For comparison, SICM topographical data of the same NPs yielded an average value of $d_{\mathrm{NP}}=136 \pm 22 \mathrm{~nm}$ 
A
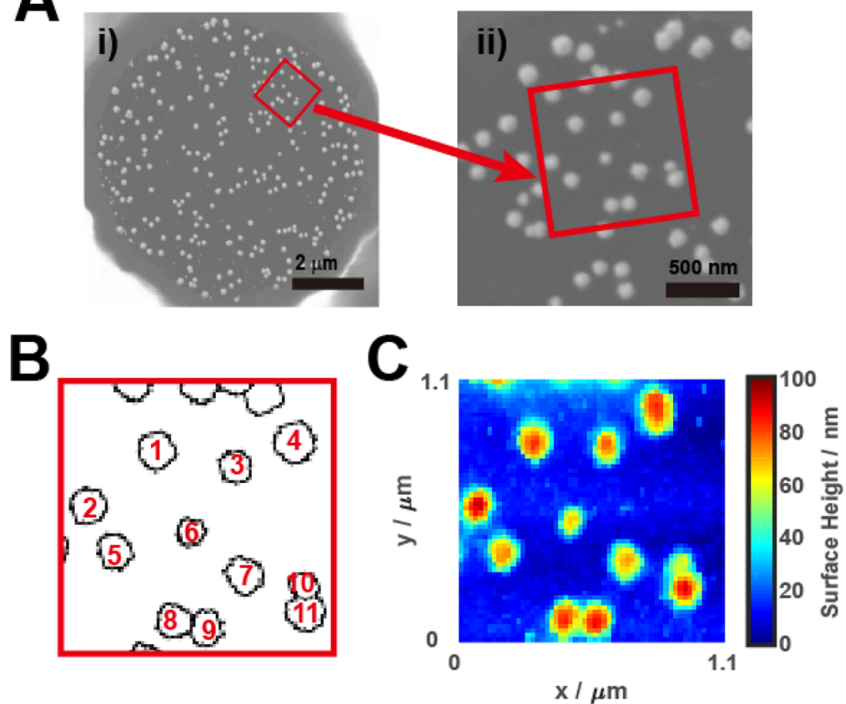

Figure 5. (A) SEM images of AuNPs on a CF UME: (i) full picture of the CF UME with AuNPs and (ii) magnified view of the red box in (i), indicating the area scanned with SICM. (B) Annotation of individual NPs from the SEM map and (C) corresponding topographical SICM data (pixel density: 2600 pixels $\mu \mathrm{m}^{-2}$ ) in a solution containing $3 \mathrm{mM} \mathrm{NaBH}$ and $30 \mathrm{mM} \mathrm{NaOH}$ at a $V_{\text {tip }}$ of $+0.15 \mathrm{~V}$. There is no interpolation of the data, and the SICM image contains 3136 pixels.

and $h_{\mathrm{NP}}=72 \pm 11 \mathrm{~nm}(N=11)$ (Figure 5C), in excellent agreement with electron microscopy. Further, the $d_{\mathrm{NP}}$ values from electron microscopy within the scanned area were individually compared NP by NP with the results from SICM (see Supporting Information, Table S2), and on average, the values derived from the SICM data were only slightly larger than those determined by electron microscopy. It is worth highlighting that the spatial resolution achievable by SICM is such that closely spaced adjacent NPs, as shown by Au NPs "8" and " 9 " as well as " 10 " and " 11 " (Figure $5 \mathrm{~B}, \mathrm{C}$ ), are readily resolved.
Figure 6 shows topography (i) and activity (ii) images obtained at $V_{\text {sub2 }}$ values of (A) $0.65 \mathrm{~V}$, (B) $0.75 \mathrm{~V}$, (C) $0.9 \mathrm{~V}$, and (D) $0.95 \mathrm{~V}$. Values of $V_{\text {sub2 }}$ were chosen to span a range of activity, deduced by measuring a $\mathrm{CV}\left(3 \mathrm{mM} \mathrm{NaBH}_{4}\right.$ in $30 \mathrm{mM}$ $\mathrm{NaOH}$ aqueous solution) at the same CF UME with adhered AuNPs (see Supporting Information, Figure S9). Although there is a small amount of drift between each image, it is clear that a similar area is scanned at each $V_{\text {sub2 }}$ and that there is good agreement between the topographical and electrochemical activity maps (pixel density was 2600 pixel $\mu \mathrm{m}^{-2}$ ) which pinpoint the NPs in different ways.

Considering the diffusion coefficient of $\mathrm{BH}_{4}{ }^{-}\left(D_{\mathrm{BH}_{4}}{ }^{-}=1.6 \times\right.$ $\left.10^{-5} \mathrm{~cm}^{2} \mathrm{~s}^{-1}\right)^{50}$ and that $I_{\text {tip }}$ values are the average of the last 5 $\mathrm{ms}$ of a $20 \mathrm{~ms} V_{\text {sub2 }}$ pulse, the corresponding diffusion length is ca. $6-8 \mu \mathrm{m}\left[\approx(2 D t)^{1 / 2}\right] .^{63}$ This is on the order of the diameter of the UME substrate, which as a rule of thumb is a reasonable approximation for the characteristic diffusion layer thickness under steady-state conditions. On this time scale, the diffusionlimited current at a UME of this size is only about $20 \%$ higher than the true steady-state value. ${ }^{64}$ There is thus significant overlap between the concentration boundary layers of neighboring NPs. Nonetheless, contrast between individual AuNPs can still be observed in the activity images, especially those obtained at lower overpotentials, due to the uneven particle distribution over the CF surface (see Figure 4A,B). Toy simulations of arrays of NPs, with varying separation distances (see Supporting Information, section S8), bear out the expectation: for the same NP reaction kinetics, with arrays of particles that are more bunched (higher NP density), a stronger depletion of ionic reactants occurs, and hence a lower normalized $I_{\text {tip }}$ is expected, as is seen in Figure 6.

It should also be noted that at applied potentials of 0.9 and $0.95 \mathrm{~V}$, the AuNPs annotated as "10" and "11" (Figure 5B) were seen to have a distinctly high apparent electrochemical activity (i.e., low measured normalized $I_{\text {tip }}$ ) when compared to that of the other AuNPs ("1-9"). It is unlikely this is simply attributable to their size (see Supporting Information, section S8), as similarly sized and spaced Au NPs "8" and "9", adjacent to each other, did not show such an enhancement of activities.
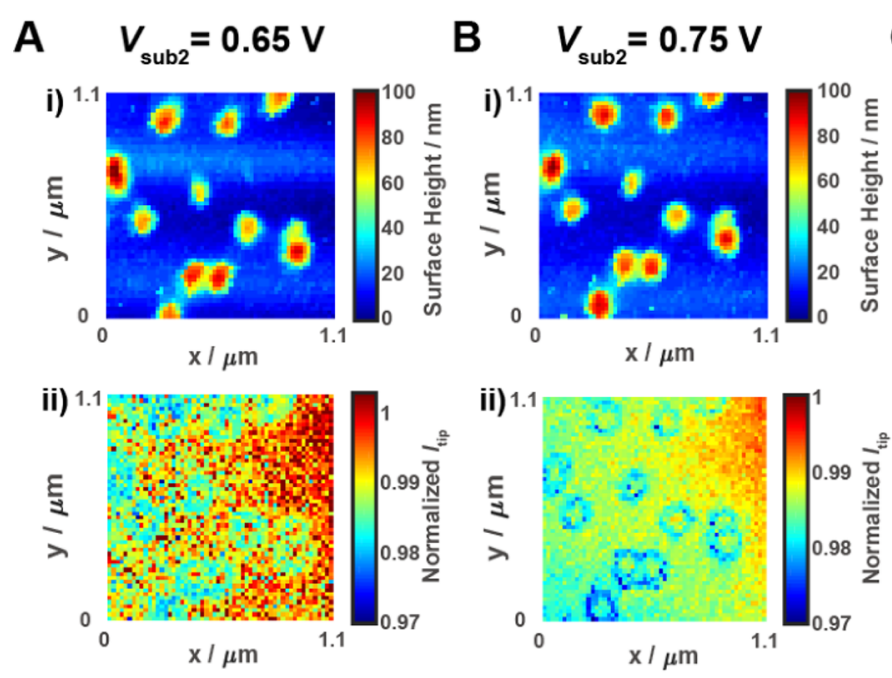
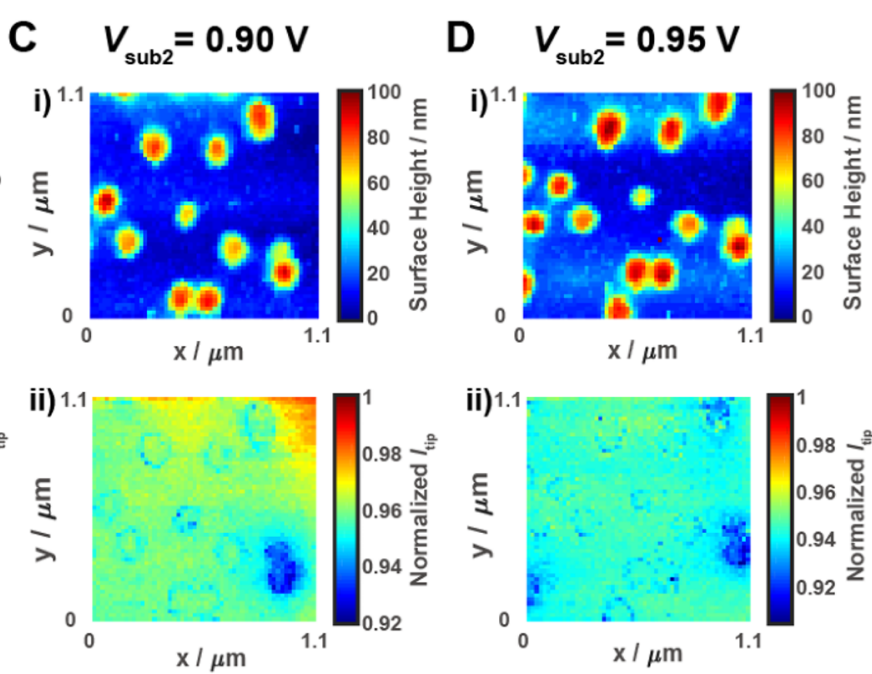

Figure 6. (i) SICM topography maps and (ii) simultaneously recorded activity images of AuNPs on a CF UME $($ diameter $=7 \mu \mathrm{m})($ pixel density: 2600 pixels $\left.\mu \mathrm{m}^{-2}\right)$, obtained in a solution containing $3 \mathrm{mM} \mathrm{NaBH}_{4}$ and $30 \mathrm{mM} \mathrm{NaOH}$, at $V_{\text {sub2 }}$ values of (A) $0.65 \mathrm{~V},(\mathrm{~B}) 0.75 \mathrm{~V},(\mathrm{C})$ $0.9 \mathrm{~V}$, and (D) $0.95 \mathrm{~V}$. During mapping, $V_{\text {tip }}$ and $d_{\text {s-t }}$ were fixed at $+0.15 \mathrm{~V}$ and $26 \mathrm{~nm}$, respectively. There is no interpolation of the data, and the images contain 3136 pixels. 
As the electro-oxidation of $\mathrm{BH}_{4}^{-}$on the $\mathrm{Au}$ is surface-sensitive, it is possible that surface sites that are more favorable to the reaction are more exposed on particles " 10 " and " 11 " (i.e., there is a legitimate difference in electrocatalytic activity). ${ }^{7,52}$ In the future, it could be interesting to further resolve the crystallographic structure/orientation of these surfaces, for example, using in situ electrochemical STM, ${ }^{32}$ although such measurements are challenging, or ex situ using selected area electron diffraction of well-defined crystalline NPs. ${ }^{65}$

At the lower values of applied $V_{\text {sub2 }}$ [Figure 6A,B(ii)], there is a noticeable ring shape of lower tip current around the NPs than on the NP top surfaces. To understand this prominent effect, FEM simulations were performed on a single NP, assuming a uniform rate constant (and a first-order reaction in both $\left[\mathrm{OH}^{-}\right]$and $\left[\mathrm{BH}_{4}^{-}\right]$for simplicity) over the NP surface (assumed to be ellipsoidal). Full details of the performed simulations are presented in the Supporting Information, section S3, and some of the key results are shown in Figure 7. Figure 7A,B presents profiles of the total ionic concentration around a NP at slower and faster kinetics, respectively.
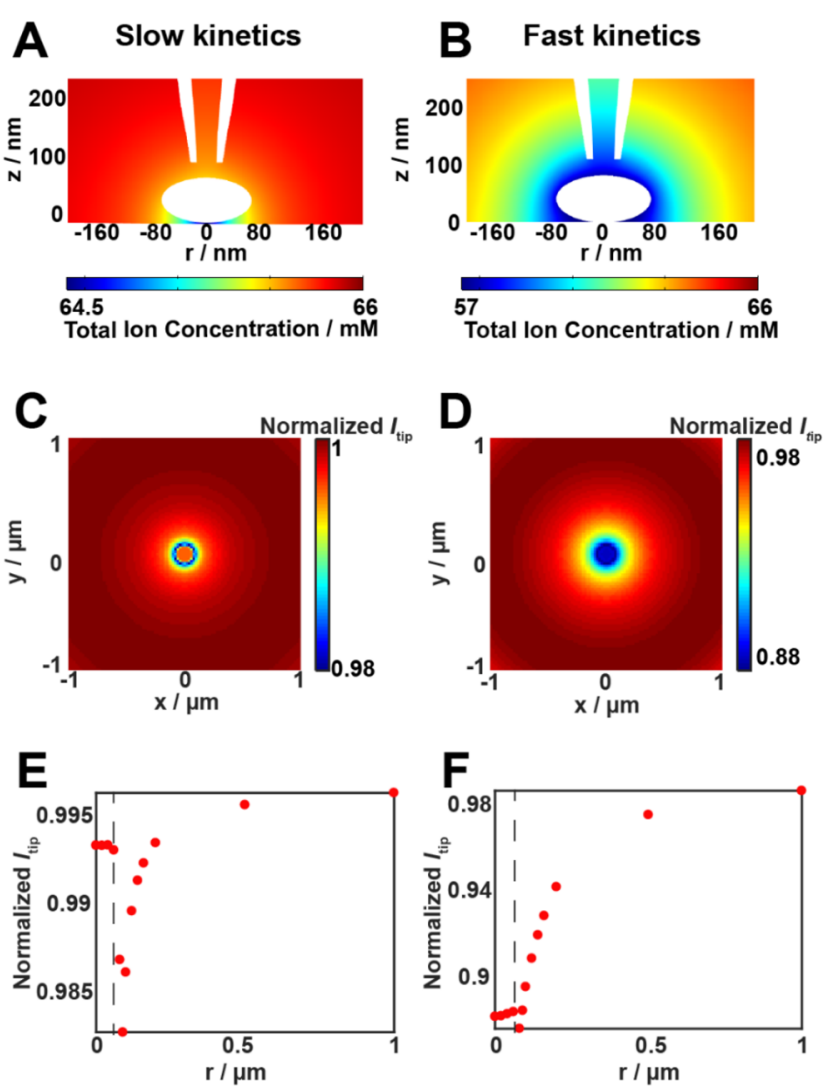

Figure 7. FEM simulations of an isolated NP with $\mathrm{BH}_{4}^{-}$oxidation at two different heterogeneous rate constants $(k)$, i.e., $3.4 \times 10^{3} \mathrm{~cm}^{4}$ $\mathrm{mol}^{-1} \mathrm{~s}^{-1}$ (low) and $1.2 \times 10^{6} \mathrm{~cm}^{4} \mathrm{~mol}^{-1} \mathrm{~s}^{-1}$ (high). Total ion concentration profiles ( $A$ and $B$ ) showing how the ion concentration around a NP (and at the end of the tip) changes with the reaction rate. (C,D) Predicted tip current profiles around the NP showing the same ring effect observed experimentally at low $k$, in particular. (E,F) Simulated tip current versus radial direction profiles with the nanopipette tracing the NP or substrate at a distance corresponding the approach current threshold and the small lift-up (i.e., $26 \mathrm{~nm}$ when over the particle center) at low and high $k$, respectively. $(\mathrm{C}-\mathrm{F})$ "Ring effect" in the activity around the $\mathrm{NP}$, notably at low $k$.
Analogous to what is seen experimentally (Figure 6), there is an increased depletion of the local ion concentration observed as the system switches from low to high driving force, which would be manifested as a decreased normalized $I_{\text {tip }}$ as seen experimentally (Figure 6).

Notably, when the kinetics of $\mathrm{BH}_{4}^{-}$oxidation are slow at the NP (Figure 7A), there is more depletion of ionic reactants in the narrow gap between the NP and the surface, compared to that with the top surface of the NPs, as also seen experimentally at lower potentials (i.e., the ring shapes in the electrochemical activity maps in Figure 6). The ring effect is less apparent at higher driving force as depletion around the entire particle becomes more significant (Figure 7B,D,F), as also seen experimentally (Figure 6). The effect observed around the NP at lower overpotential is a mass transport/geometry effect: the narrow gap between the NP and surface restricts mass transport, and so there is more depletion of reactants. These results provide direct visual demonstration supporting previous studies of similar effects proposed for hierarchically structured nanomaterials ${ }^{66-68}$ and serve to highlight major perspectives on the different overall activity that prevails on different parts of a NP.

\section{CONCLUSIONS}

We have introduced a procedure for electrochemical (reaction) imaging at the nanoscale using a simple single-channel SICM tip. Through careful control of the substrate potential-time profile and continuous recording of the SICM tip current at high frequency during the imaging process, it is possible to map topography and electrochemical activity simultaneously. The small nanopipette probe (30 nm diameter used herein) has enabled high-resolution imaging at the subparticle level, revealing how reactive flux is distributed among groups of NPs depending on the spacing and intrinsic NP activity. An important feature observed in this work, which has not been seen previously, is that the geometry of the nanomaterial with respect to the substrate (for example, the narrow gap formed between AuNPs and the CF support compared to the more accessible NP top surface) plays an important role in the distribution of ion fluxes around reactive nanoentities, most noticeable at low overpotentials. These issues have been explored and explained with FEM simulations that support the experimental results.

The studies herein demonstrate SICM as an important technique to study electrochemical activity and ion fluxes and the correlation of activity to the structure and morphology of nanomaterials. One could envisage future wide applications to study nanomaterials used for rechargeable/renewable energy and in electrochemical sensors, among other applications. The SICM regime described herein is easily implemented and has a number of advantages compared to other SEPMs, not least (i) a very simple tip fabrication and characterization procedure; (ii) a tip response that is very stable and whose status is checked at each and every pixel (and self-referenced); and (iii) the possibility of obtaining images on a reasonably fast time scale.

\section{METHODS}

Chemicals. Sodium borohydride $\left(\mathrm{NaBH}_{4}, 99.99 \%\right.$, Sigma-Aldrich), sodium hydroxide $(\mathrm{NaOH},>97 \%$, Fisher Scientific), perchloric acid $\left(\mathrm{HClO}_{4}, 70 \%\right.$, ACS reagent, Sigma-Aldrich), and chloroauric acid trihydrate $\left(\mathrm{HAuCl}_{4} \cdot 3 \mathrm{H}_{2} \mathrm{O}, \geq 99.9 \%\right.$, Sigma-Aldrich) were used as provided by the supplier. All solutions were prepared with Milli-Q reagent grade water (resistivity ca. $18.2 \mathrm{M} \Omega \cdot \mathrm{cm}$ at $25{ }^{\circ} \mathrm{C}$ ). Electrodeposition of Au nanostructures was carried out with $0.5 \mathrm{mM}$ 
$\mathrm{HAuCl}_{4}$ in $0.1 \mathrm{M} \mathrm{HClO}_{4}$ aqueous solution. SICM electrochemical imaging was carried out with $3 \mathrm{mM} \mathrm{NaBH}_{4}$ in $30 \mathrm{mM} \mathrm{NaOH}$ aqueous solution.

Nanopipette and Sample Preparation. Nanopipettes (diameter $\approx 30 \mathrm{~nm}$ ) were fabricated from quartz capillaries $(1 \mathrm{~mm}$ o.d., $0.5 \mathrm{~mm}$ i.d., Friedrich \& Dimmock) using a laser puller (P-2000, Sutter Instruments) and characterized by TEM (Jeol 2000X, HT $=200 \mathrm{eV}$ ), after SICM measurements. Two different types of UME sealed in a glass sheath, $\mathrm{CF}$ (diameter $=7 \mu \mathrm{m}$, XAS grade, Goodfellow) and $\mathrm{Au}$ (diameter $=10 \mu \mathrm{m}, 99.99+\%$, Goodfellow), were prepared as substrates. UMEs were polished with a microcloth (Buehler) and immersed in alumina powder solution (MicroPolish Alumina, $0.05 \mu \mathrm{m}$, Buehler) followed by a mechanical polishing step using diamond lapping films (UltraPrep Diamond Lapping Films, $0.1 \mu \mathrm{m}$, Buehler). CF UMEs were further used as a supporting substrate for electrodeposited $\mathrm{Au}$ nanostructures or $\mathrm{Au}$ nanoparticles. $\mathrm{Au}$ nanostructures were prepared by applying a constant potential of 0.5 $\mathrm{V} v s \mathrm{Ag} / \mathrm{AgCl}$ for $14 \mathrm{~s}$ to the CF UME in $0.5 \mathrm{mM} \mathrm{HAuCl}_{4}$ with $0.1 \mathrm{M}$ $\mathrm{HClO}_{4}$, and AuNPs were prepared by applying $0 \mathrm{~V} v \mathrm{sg} / \mathrm{AgCl}$ for $1 \mathrm{~s}$ to the CF UME, in the same plating solution. The size and shape of the nanostructured $\mathrm{Au}$ on the CF UMEs was characterized with SEM (Zeiss Sigma FE-SEM, Carl Zeiss AG). Sample preparation for crosssectional analysis of the AuNPs was performed using focused-ion beam-SEM (Scios DualBeam, FEI), and the sample was characterized with TEM (Talos F200X, FEI).

Scanning Ion Conductance Microscopy. A quartz nanopipette was mounted on a custom-designed holder and positioned over the area of interest using a mechanical micropositioner (Newport, M-461XYZ-M) and a 3MP digital camera (PixelLink, PL-B776U) with a $6 \times$ magnification lens. Vertical coarse movement of the nanopipette was achieved with a picomotor (Newport, 8303 Picomotor Actuator), utilized to bring the nanopipette within the travel range of the singleaxis nanopositioner. A $15 \mu \mathrm{m}$ range single-axis nanopositioner (Physik Instrumente, P-753.1CD; positional error $=0.05 \mathrm{~nm}$ ) was used for precise vertical movement of the nanopipette with the protocol described in the main text, with fine lateral moment of the sample achieved using a highly precise $X Y$ pieozoelectric stage (Physik Instrumente, P-733.2DD). The SICM system was installed on an optical table (Newport, RS2000) equipped with automatic leveling isolators (Newport, S-2000A-423.5).

A DC feedback hopping mode was used. ${ }^{39,69}$ A Faraday cage enclosed the SICM system with vacuum insulation panels (Kevothermal) and aluminum heat sinks to reduce thermal drift of the piezoelectric positioners. A home-built potentiostat and electrometer were used for electrochemical measurements. Two Pd wires saturated with hydrogen $\left(\mathrm{Pd}-\mathrm{H}_{2}\right)$, prepared as reported elsewhere, ${ }^{25,54,70}$ served as quasi-reference counter electrodes. Control of instrumentation and data acquisition was conducted with a customdeveloped LabVIEW (2016, National Instruments) program through an FPGA card (NI PCIe-7852e). Data were acquired at a rate of $c a$. $516 \mu$ s per point (resulting from a $4 \mu$ s sampling time and averaging of 129 sample points). The nanopipette approached the surface at a rate of $3 \mu \mathrm{m} \mathrm{s}^{-1}$ at each pixel until the desired set point of $I_{\text {tip }}$ was achieved (i.e., a decrease of $4.1 \%$ between the bulk and surface, herein) and immediately pulled back to the desired distance. After being held in this position for $40 \mathrm{~ms}$, during which time the substrate potential was switched, the tip was retracted away from the surface and moved to the next pixel, where the process was repeated.

FEM Simulations. FEM simulations were performed in COMSOL Multiphysics v5.2a. Details are presented in Supporting Information, section S3. Simulations encompassed a 2D axisymmetric representation of the nanopipette, with dimensions extracted from TEM images, to extract the surface charge value of the glass walls. 3D simulations of the nanopipette geometry above a typical ellipsoidal NP geometry were performed with approach curves being generated to estimate the pipette-surface working distance that was achieved experimentally. Approach curves were simulated at different positions along a surface containing a NP to determine if the approach distance achieved in the experiments was relatively consistent regardless of the tip position with respect to the NP. Models with a reactive substrate were also covered.

\section{ASSOCIATED CONTENT}

\section{Supporting Information}

The Supporting Information is available free of charge on the ACS Publications website at DOI: 10.1021/acsnano.7b05435.

Nanopipette and $\mathrm{Au} \mathrm{NP}$ characterizations, a typical $I_{\text {tip }}$ trace in a single hop, details of FEM simulations, $I_{\text {tip }}$ sensitivity on $V_{\text {tip }}, V_{\text {sub2 }}$, and materials, a $\mathrm{CV}$ of $\mathrm{BH}_{4}^{-}$ oxidation on Au NPs on CF UME, and FEM simulations for effect of NP spacing (PDF)

\section{AUTHOR INFORMATION}

\section{Corresponding Author}

*E-mail: p.r.unwin@warwick.ac.uk.

ORCID ब

Minkyung Kang: 0000-0003-3248-8496

David Perry: 0000-0002-8630-4798

Cameron L. Bentley: 0000-0001-7867-6068

Ashley Page: 0000-0001-7233-6337

Patrick R. Unwin: 0000-0003-3106-2178

\section{Notes}

The authors declare no competing financial interest.

\section{ACKNOWLEDGMENTS}

The work was supported by the University of Warwick Chancellor's International Scholarship (M.K.). D.P. thanks the Leverhulme Trust for support via a research project grant. C.L.B. and A.P. acknowledge support from a Marie Curie Individual Fellowship (702048 NEIL) and the EPSRC through the MOAC DTC (EP/F500378/1), respectively. The authors thank Dmitry Momotenko for helpful discussions for initial setup of the experiments.

\section{REFERENCES}

(1) Perreault, F.; Fonseca de Faria, A.; Elimelech, M. Environmental Applications of Graphene-Based Nanomaterials. Chem. Soc. Rev. 2015, $44,5861-5896$

(2) Chen, A.; Chatterjee, S. Nanomaterials Based Electrochemical Sensors for Biomedical Applications. Chem. Soc. Rev. 2013, 42, 54255438.

(3) Trogadas, P.; Ramani, V.; Strasser, P.; Fuller, T. F.; Coppens, M. O. Hierarchically Structured Nanomaterials for Electrochemical Energy Conversion. Angew. Chem., Int. Ed. 2016, 55, 122-148.

(4) Raccichini, R.; Varzi, A.; Passerini, S.; Scrosati, B. The Role of Graphene for Electrochemical Energy Storage. Nat. Mater. 2015, 14, 271-279.

(5) Kleijn, S. E. F.; Lai, S. C. S.; Koper, M. T. M.; Unwin, P. R. Electrochemistry of Nanoparticles. Angew. Chem., Int. Ed. 2014, 53, $3558-3586$.

(6) Wu, J.; Yang, H. Platinum-Based Oxygen Reduction Electrocatalysts. Acc. Chem. Res. 2013, 46, 1848-1857.

(7) Rodriguez, P.; Koper, M. T. M. Electrocatalysis on Gold. Phys. Chem. Chem. Phys. 2014, 16, 13583-13594.

(8) Sambur, J. B.; Chen, P. Approaches to Single-Nanoparticle Catalysis. Annu. Rev. Phys. Chem. 2014, 65, 395-422.

(9) Oja, S. M.; Fan, Y.; Armstrong, C. M.; Defnet, P.; Zhang, B. Nanoscale Electrochemistry Revisited. Anal. Chem. 2016, 88, 414430

(10) Xiao, X.; Bard, A. J. Observing Single Nanoparticle Collisions at an Ultramicroelectrode by Electrocatalytic Amplification. J. Am. Chem. Soc. 2007, 129, 9610-9612.

(11) Li, Y.; Cox, J. T.; Zhang, B. Electrochemical Responses and Electrocatalysis at Single Au Nanoparticles. J. Am. Chem. Soc. 2010, $132,3047-3054$. 
(12) Wilson, A. J.; Willets, K. A. Visualizing Site-Specific Redox Potentials on the Surface of Plasmonic Nanoparticle Aggregates with Superlocalization SERS Microscopy. Nano Lett. 2014, 14, 939-945.

(13) Sambur, J. B.; Chen, T.-Y.; Choudhary, E.; Chen, G.; Nissen, E. J.; Thomas, E. M.; Zou, N.; Chen, P. Sub-Particle Reaction and Photocurrent Mapping to Optimize Catalyst-Modified Photoanodes. Nature 2016, 530, 77-80.

(14) Willets, K. A.; Wilson, A. J.; Sundaresan, V.; Joshi, P. B. SuperResolution Imaging and Plasmonics. Chem. Rev. 2017, 117, 75387582 .

(15) Kang, M.; Momotenko, D.; Page, A.; Perry, D.; Unwin, P. R. Frontiers in Nanoscale Electrochemical Imaging: Faster, Multifunctional, and Ultrasensitive. Langmuir 2016, 32, 7993-8008.

(16) Actis, P.; Tokar, S.; Clausmeyer, J.; Babakinejad, B.; Mikhaleva, S.; Cornut, R.; Takahashi, Y.; Lopez Cordoba, A.; Novak, P.; Shevchuck, A. I.; et al. Electrochemical Nanoprobes for Single-Cell Analysis. ACS Nano 2014, 8, 875-884.

(17) Thakar, R.; Weber, A. E.; Morris, C. A.; Baker, L. A. Multifunctional Carbon Nanoelectrodes Fabricated by Focused Ion Beam Milling. Analyst 2013, 138, 5973-5982.

(18) Blanchard, P. Y.; Sun, T.; Yu, Y.; Wei, Z.; Matsui, H.; Mirkin, M. V. Scanning Electrochemical Microscopy Study of Permeability of a Thiolated Aryl Multilayer and Imaging of Single Nanocubes Anchored to It. Langmuir 2016, 32, 2500-2508.

(19) Yu, Y.; Sun, T.; Mirkin, M. V. Scanning Electrochemical Microscopy of Single Spherical Nanoparticles: Theory and Particle Size Evaluation. Anal. Chem. 2015, 87, 7446-7453.

(20) Kim, J.; Renault, C.; Nioradze, N.; Arroyo-Currás, N.; Leonard, K. C.; Bard, A. J. Electrocatalytic Activity of Individual Pt Nanoparticles Studied by Nanoscale Scanning Electrochemical Microscopy. J. Am. Chem. Soc. 2016, 138, 8560-8568.

(21) Unwin, P. R.; Güell, A. G.; Zhang, G. Nanoscale Electrochemistry of $\mathrm{sp}^{2}$ Carbon Materials: From Graphite and Graphene to Carbon Nanotubes. Acc. Chem. Res. 2016, 49, 2041-2048.

(22) Lai, S. C. S.; Dudin, P. V.; MacPherson, J. V.; Unwin, P. R. Visualizing Zeptomole (Electro)catalysis at Single Nanoparticles within an Ensemble. J. Am. Chem. Soc. 2011, 133, 10744-10747.

(23) Momotenko, D.; Byers, J. C.; McKelvey, K.; Kang, M.; Unwin, P. R. High-Speed Electrochemical Imaging. ACS Nano 2015, 9, 89428952.

(24) Güell, A. G.; Meadows, K. E.; Dudin, P. V.; Ebejer, N.; Macpherson, J. V.; Unwin, P. R. Mapping Nanoscale Electrochemistry of Individual Single-Walled Carbon Nanotubes. Nano Lett. 2014, 14, 220-224.

(25) Chen, C. H.; Jacobse, L.; McKelvey, K.; Lai, S. C. S.; Koper, M. T. M.; Unwin, P. R. Voltammetric Scanning Electrochemical Cell Microscopy: Dynamic Imaging of Hydrazine Electro-Oxidation on Platinum Electrodes. Anal. Chem. 2015, 87, 5782-5789.

(26) Kranz, C.; Friedbacher, G.; Mizaikoff, B.; Lugstein, A.; Smoliner, J.; Bertagnolli, E. Integrating an Ultramicroelectrode in an AFM Cantilever: Combined Technology for Enhanced Information. Anal. Chem. 2001, 73, 2491-2500.

(27) Macpherson, J. V.; Unwin, P. R. Combined Scanning Electrochemical-Atomic Force Microscopy. Anal. Chem. 2000, 72, 276-285.

(28) O'Connell, M. A.; Lewis, J. R.; Wain, A. J. Electrochemical Imaging of Hydrogen Peroxide Generation at Individual Gold Nanoparticles. Chem. Commun. 2015, 51, 10314-10317.

(29) Page, A.; Kang, M.; Armitstead, A.; Perry, D.; Unwin, P. R. Quantitative Visualization of Molecular Delivery and Uptake at Living Cells with Self-Referencing Scanning Ion Conductance Microscopy (SICM) - Scanning Electrochemical Microscopy (SECM). Anal. Chem. 2017, 89, 3021-3028.

(30) Takahashi, Y.; Shevchuk, A. I.; Novak, P.; Zhang, Y.; Ebejer, N.; MacPherson, J. V.; Unwin, P. R.; Pollard, A. J.; Roy, D.; Clifford, C. A.; Shiku, H.; Matsue, T.; Klenerman, D.; Korchev, Y. E. Multifunctional Nanoprobes for Nanoscale Chemical Imaging and Localized Chemical Delivery at Surfaces and Interfaces. Angew. Chem., Int. Ed. 2011, 50, 9638-9642.
(31) Sklyar, O.; Treutler, T. H.; Vlachopoulos, N.; Wittstock, G. The Geometry of Nanometer-Sized Electrodes and Its Influence on Electrolytic Currents and Metal Deposition Processes in Scanning Tunneling and Scanning Electrochemical Microscopy. Surf. Sci. 2005, 597, 181-195.

(32) Meier, J.; Friedrich, K. A.; Stimming, U. Novel Method for the Investigation of Single Nanoparticle Reactivity. Faraday Discuss. 2002, $121,365-372$.

(33) Takahashi, Y.; Shevchuk, A. I.; Novak, P.; Babakinejad, B.; Macpherson, J.; Unwin, P. R.; Shiku, H.; Gorelik, J.; Klenerman, D.; Korchev, Y. E.; Matsue, T. Topographical and Electrochemical Nanoscale Imaging of Living Cells Using Voltage-Switching Mode Scanning Electrochemical Microscopy. Proc. Natl. Acad. Sci. U. S. A. 2012, 109, 11540-11545.

(34) Kranz, C. Recent Advancements in Nanoelectrodes and Nanopipettes Used in Combined Scanning Electrochemical Microscopy Techniques. Analyst 2014, 139, 336-352.

(35) Novak, P.; Shevchuk, A.; Ruenraroengsak, P.; Miragoli, M.; Thorley, A. J.; Klenerman, D.; Lab, M. J.; Tetley, T. D.; Gorelik, J.; Korchev, Y. E. Imaging Single Nanoparticle Interactions with Human Lung Cells Using Fast Ion Conductance Microscopy. Nano Lett. 2014, 14, 1202-1207.

(36) Lipson, A. L.; Ginder, R. S.; Hersam, M. C. Nanoscale in Situ Characterization of Li-Ion Battery Electrochemistry via Scanning Ion Conductance Microscopy. Adv. Mater. 2011, 23, 5613-5617.

(37) Lipson, A. L.; Puntambekar, K.; Comstock, D. J.; Meng, X.; Geier, M. L.; Elam, J. W.; Hersam, M. C. Nanoscale Investigation of Solid Electrolyte Interphase Inhibition on Li-Ion Battery $\mathrm{MnO}$ Electrodes via Atomic Layer Deposition of $\mathrm{Al}_{2} \mathrm{O}_{3}$. Chem. Mater. 2014, 26, 935-940.

(38) Chen, C.-C.; Zhou, Y.; Baker, L. A. Scanning Ion Conductance Microscopy. Annu. Rev. Anal. Chem. 2012, 5, 207-228.

(39) Page, A.; Perry, D.; Young, P.; Mitchell, D. A.; Frenguelli, B. G.; Unwin, P. R. Fast Nanoscale Surface Charge Mapping with PulsedPotential Scanning Ion Conductance Microscopy. Anal. Chem. 2016, $88,10854-10859$.

(40) Perry, D.; Paulose Nadappuram, B.; Momotenko, D.; Voyias, P. D.; Page, A.; Tripathi, G.; Frenguelli, B. G.; Unwin, P. R. Surface Charge Visualization at Viable Living Cells. J. Am. Chem. Soc. 2016, $138,3152-3160$

(41) Ida, H.; Takahashi, Y.; Kumatani, A.; Shiku, H.; Matsue, T. High Speed Scanning Ion Conductance Microscopy for Quantitative Analysis of Nanoscale Dynamics of Microvilli. Anal. Chem. 2017, 89, 6015.

(42) Page, A.; Perry, D.; Unwin, P. R. Multifunctional Scanning Ion Conductance Microscopy. Proc. R. Soc. London, Ser. A 2017, 473, 20160889.

(43) McKelvey, K.; Kinnear, S. L.; Perry, D.; Momotenko, D.; Unwin, P. R. Surface Charge Mapping with a Nanopipette. J. Am. Chem. Soc. 2014, 136, 13735-13744.

(44) Momotenko, D.; McKelvey, K.; Kang, M.; Meloni, G. N.; Unwin, P. R. Simultaneous Interfacial Reactivity and Topography Mapping with Scanning Ion Conductance Microscopy. Anal. Chem. 2016, 88, 2838-2846.

(45) Bode, D. D.; Andersen, T. N.; Eyring, H. Anion and pH Effects on the Potentials of Zero Charge of Gold and Silver Electrodes. J. Phys. Chem. 1967, 71, 792-797.

(46) Gyenge, E. Electrooxidation of Borohydride on Platinum and Gold Electrodes: Implications for Direct Borohydride Fuel Cells. Electrochim. Acta 2004, 49, 965-978.

(47) Atwan, M. H.; Macdonald, C. L. B.; Northwood, D. O.; Gyenge, E. L. Colloidal Au and Au-Alloy Catalysts for Direct Borohydride Fuel Cells: Electrocatalysis and Fuel Cell Performance. J. Power Sources 2006, 158, 36-44.

(48) Ida, H.; Takahashi, Y.; Kumatani, A.; Shiku, H.; Matsue, T. High Speed Scanning Ion Conductance Microscopy for Quantitative Analysis of Nanoscale Dynamics of Microvilli. Anal. Chem. 2017, 89, 6015-6020. 
(49) Gardiner, J. A.; Collat, J. W. Kinetics of the Stepwise Hydrolysis of Tetrahydroborate Ion. J. Am. Chem. Soc. 1965, 87, 1692-1700.

(50) Mirkin, M. V.; Yang, H.; Bard, A. J. Borohydride Oxidation at a Gold Electrode. J. Electrochem. Soc. 1992, 139, 2212-2217.

(51) Pasqualeti, A. M.; Olu, P. Y.; Chatenet, M.; Lima, F. H. B. Borohydride Electrooxidation on Carbon-Supported Noble Metal Nanoparticles: Insights into Hydrogen and Hydroxyborane Formation. ACS Catal. 2015, 5, 2778-2787.

(52) Escaño, M. C. S.; Arevalo, R. L.; Gyenge, E.; Kasai, H. Electrocatalysis of Borohydride Oxidation: A Review of Density Functional Theory Approach Combined with Experimental Validation. J. Phys.: Condens. Matter 2014, 26, 353001.

(53) Iotov, P. I.; Kalcheva, S. V.; Bond, A. M. Kinetic and Mechanistic Evaluation of Tetrahydroborate Ion Electro-Oxidation at Polycrystalline Gold. Electrochim. Acta 2009, 54, 7236-7241.

(54) Bentley, C. L.; Kang, M.; Unwin, P. R. Time-Resolved Detection of Surface Oxide Formation at Individual Gold Nanoparticles: Role in Electrocatalysis and New Approach for Sizing by Electrochemical Impacts. J. Am. Chem. Soc. 2016, 138, 12755-12758.

(55) Burke, L. D. Scope for New Applications for Gold Arising from the Electrocatalytic Behaviour of Its Metastable Surface States. Gold Bull. 2004, 37, 125-135.

(56) Momotenko, D.; Page, A.; Adobes-Vidal, M.; Unwin, P. R. Write-Read 3D Patterning with a Dual-Channel Nanopipette. ACS Nano 2016, 10, 8871-8878.

(57) Perry, D.; Momotenko, D.; Lazenby, R. A.; Kang, M.; Unwin, P. R. Characterization of Nanopipettes. Anal. Chem. 2016, 88, 55235530.

(58) Sa, N.; Baker, L. A. Rectification of Nanopores at Surfaces. J. Am. Chem. Soc. 2011, 133, 10398-10401.

(59) Sa, N.; Lan, W. J.; Shi, W.; Baker, L. A. Rectification of Ion Current in Nanopipettes by External Substrates. ACS Nano 2013, 7, $11272-11282$.

(60) Burt, D. P.; Wilson, N. R.; Janus, U.; Macpherson, J. V.; Unwin, P. R. In-Situ Atomic Force Microscopy (AFM) Imaging: Influence of AFM $\backslash$ rProbe Geometry on Diffusion to Microscopic Surfaces. Langmuir 2008, 24, 12867-12876.

(61) Matsushima, H.; Lin, S.-W.; Morin, S.; Magnussen, O. M. In Situ Video-STM Studies of the Mechanisms and Dynamics of Electrochemical Bismuth Nanostructure Formation on Au. Faraday Discuss. 2016, 193, 171-185.

(62) Dong, H.; Feng, R.; Ai, X.; Cao, Y.; Yang, H.; Cha, C. Electrooxidation Mechanisms and Discharge Characteristics of Borohydride on Different Catalytic Metal Surfaces. J. Phys. Chem. B 2005, 109, 10896-10901.

(63) Bard, A. J.; Faulkner, L. R. Electrochemical Methods: Fundamentals and Applications, 2nd ed.; Wiley: New York, 2001.

(64) Shoup, D.; Szabo, A. Chronoamperometric Current at Finite Disk Electrodes. J. Electroanal. Chem. Interfacial Electrochem. 1982, 140, 237-245.

(65) Qin, Y.; Zhang, X.; Dai, X.; Sun, H.; Yang, Y.; Li, X.; Shi, Q.; Gao, D.; Wang, H.; Yu, N. F.; Sun, S. G. Graphene Oxide-Assisted Synthesis of Pt-Co Alloy Nanocrystals with High-Index Facets and Enhanced Electrocatalytic Properties. Small 2016, 12, 524-533.

(66) Han, J. H.; Lee, E.; Park, S.; Chang, R.; Chung, T. D. Effect of Nanoporous Structure on Enhanced Electrochemical Reaction. J. Phys. Chem. C 2010, 114, 9546-9553.

(67) Bae, J.; Han, J.; Chung, T. Electrochemistry at Nanoporous Interfaces: New Opportunity for Electrocatalysis. Phys. Chem. Chem. Phys. 2012, 14, 448-463.

(68) Walcarius, A. Mesoporous Materials and Electrochemistry. Chem. Soc. Rev. 2013, 42, 4098-4140.

(69) Novak, P.; Li, C.; Shevchuk, A. I.; Stepanyan, R.; Caldwell, M.; Hughes, S.; Smart, T. G.; Gorelik, J.; Ostanin, V. P.; Lab, M. J.; Moss, G. W. J.; Frolenkov, G. I.; Klenerman, D.; Korchev, Y. E. Nanoscale Live-Cell Imaging Using Hopping Probe Ion Conductance Microscopy. Nat. Methods 2009, 6, 279-281.

(70) Chen, C.-H.; Meadows, K. E.; Cuharuc, A.; Lai, S. C. S.; Unwin, P. R. High Resolution Mapping of Oxygen Reduction Reaction
Kinetics at Polycrystalline Platinum Electrodes. Phys. Chem. Chem. Phys. 2014, 16, 18545-18552. 